\title{
Avaliação de parâmetros operacionais de um sistema de membranas para tratamento de efluentes oleosos
}

\author{
Evaluation of a membrane system's \\ operational parameters for oily effluent treatment \\ Albérico Ricardo Passos Motta** ${ }^{1 *}$, Cristiano Piacsek Borges ${ }^{2} \oplus$,
Asher Kiperstok $^{3} \odot$, Karla Esquerre $^{1} \odot$, Priscilla Maria Reis Thomé de Souza da Silva $^{4} \odot$
}

口-

\begin{abstract}
RESUMO
O objetivo deste trabalho foi discutir os principais parâmetros de controle de um sistema de membranas de microfiltração para remoção de óleo de efluentes com baseemensaios experimentais. Os ensaios utilizaram efluentes sintéticos com diâmetros de gotas de óleo entre 4 e $8 \mu \mathrm{m}$. As membranas usadas foram do tipo fibra oca com módulo submerso. Foram testados os seguintes parâmetros: concentrações de óleos e graxas na alimentação das membranas (O\&Gam) entre 100 e 260 mg.L'; taxas de recuperação de água (Rec) de 0,75 e 0,90; e pressões através das membranas $(\Delta \mathrm{p})$ de $-0,20$ e - 0,30 bar. Os resultados mostraram que as membranas produzem um permeado com uma qualidade boa a ponto de possibilitar o seu reúso em atividades industriais diversas. Foi também observado que a elevação da O\&Gam e da Rec acentuam o declínio de fluxo com o tempo e que a elevação da $\Delta$ p aumenta o valor do fluxo no tempo de seis horas.

Palavras-chave: óleo; emulsão; membranas; microfiltração.
\end{abstract}

\begin{abstract}
The aim of this paper is to discuss the main control parameters of a microfiltration membranes system for oil removal from effluent on experimental bases. The experiments were conducted by using synthetic effluent with oil droplets diameter ranging from 4 to 8 microns. The membranes used were hollow fiber-type which were assembled in a submerged module. The following parameters were tested: Oil and grease concentration in the feed stream (O\&Gf ) of 100 and 200 mg..'-1 Water recovery rates (WRR) of 0.75 and 0.90, and Transmembrane pressure (TMP) of -0.20 and -0.30 bar. The results showed that the membranes produced a permeate with a good quality to the point of enabling their reuse in several industrial activities. It was also observed that the elevation of O\&Gf and WRR increase the flux decline over time and that the increase of TMP increases the flow value in time of 6 hours.
\end{abstract}

Keywords: oil; emulsion; membranes; microfiltration.

\section{INTRODUÇÃO}

As tecnologias usualmente utilizadas para a remoção de óleo de efluentes oleosos nem sempre são capazes de atingir os níveis requeridos de eficiência (EBRAHIMI et al., 2009), principalmente se esse composto está presente sob a forma de emulsão e com diâmetros de gotas menores do que $10 \mu \mathrm{m}$ (CHAKRABARTY; GHOSHAL; PURKAIT, 2008).

O óleo pode estar presente nos efluentes sob as formas dispersa e dissolvida. Os primeiros podem ser classificados em óleo livre e emulsionado (ou em emulsão). O óleo livre está presente no efluente sob a forma de gotas com grandes diâmetros, acima de $100 \mu \mathrm{m}$, enquanto o óleo em emulsão possui diâmetro de gotas menor do que esse valor (BADER, 2007).

Existem vários métodos de aplicação industrial que atualmente são utilizados para remoção de óleo. Entre eles estão os que apresentam uma maior eficiência quando usados para a remoção de óleo livre, como os separadores gravitacionais (STEWART; ARNOLD, 2011) e hidrociclones (SAIDI et al., 2012).

Para remoção de óleo emulsionado, os métodos mais aplicados são flotação a ar precedida de coagulação química (HONG; FANE; BURFORD, 2003), coalescedores de leito (SOKOLOVIĆ; SOKOLOVIĆ; SEVIC,

'Universidade Federal da Bahia - Salvador (BA), Brasil.

'Universidade Federal do Rio de Janeiro - Rio de Janeiro (RJ), Brasil.

${ }^{3}$ Rede de Tecnologias Limpas - Salvador (BA), Brasil.

${ }^{4}$ Universidade Federal Fluminense - Rio de Janeiro (RJ), Brasil.

*Autor correspondente: arpmotta@yahoo.com.br

Recebido: 30/12/2013 - Aceito: 22/05/2018 - Reg. ABES: 128632 
2009) e filtração, esse último usando leitos de diferentes materiais, como serragem (CAMBIELLA et al., 2006), carvão ou turfa (MATHAVAN; VIRARAGHAVAN, 1992). Entretanto, esses processos usados para remoção de óleo em emulsão apresentam desvantagens em comparação com o processo de membranas. Entre elas, estão (MAITI et al., 2011):

- O uso de onerosos produtos químicos e problemas de manuseio e disposição de elevados volumes de lodo (esse é o caso da flotação a ar precedida de coagululação química);

- Nem sempre os requerimentos legais são atingidos (caso da flotação, do coalescedor de leito e da filtração);

- Elevado requerimento por área (caso da flotação);

- Baixa absorção, seletividade ineficiente para o óleo e regeneração do leito ineficiente (caso da filtração com leitos diversos), além de custos operacionais elevados (caso da filtração com leito de carvão).

Os processos de membranas podem se apresentar como uma boa solução alternativa para superar essas desvantagens, principalmente se forem utilizados como um processo compelementar, sendo precedido por um processo de remoção de óleo a montante. As suas principais vantagens são: retenção de gotas de óleo com diâmetros abaixo de $10 \mu \mathrm{m}$, o fato de dispensar a utilizacão de produtos químicos na sua operação e a capacidade de gerar permeados com qualidade aceitável (CHAKRABARTY; GHOSHAL; PURKAIT, 2008).

Entretanto, apesar do crescimento ocorrido nessa área nos últimos anos, inúmeros trabalhos de pesquisa ainda se fazem necessários a fim de que os principais obstáculos que dificultam a sua aplicação em larga escala sejam superados. Entre eles está a redução de fluxo de permeado, causada pelos fenômenos de polarizacão de concentracão e fouling (AL-OBEIDANI et al., 2008).

O objetivo deste trabalho é apresentar uma discussão sobre os principais parâmetros de controle operacional de um sistema de membranas de microfiltração para remoção de óleo emulsionado de efluentes com base em ensaios experimentais.

Para tanto, foram avaliados parâmetros diversos, como a concentração de óleo (O\&G) na corrente de alimentação das membranas (O\&Gam), a vazão de alimentação $(\mathrm{Q})$, a taxa de recuperação de água $(\operatorname{Rec})$ e a pressão através das membranas $(\Delta \mathrm{p})$. Para avaliação dos resultados, foram utilizados parâmetros ligados à qualidade do efluente final, tais como o O\&G na corrente de permeado (O\&Gp) e a eficiência percentual das membranas (Em), além de outros, como o declínio de fluxo de permeado (Jp) ao longo do tempo de operação e o valor de Jp após seis horas de operação das membranas.

\section{MATERIAIS E MÉTODOS}

Os principais dados dos materiais, equipamentos e procedimentos utilizados nos ensaios estão descritos a seguir.

\section{O efluente gerado}

Os ensaios experimentais foram realizados utilizando efluentes sintéticos produzidos, de forma contínua, por um equipamento especificamente projetado para gerar efluentes oleosos. O tipo de óleo utilizado foi petróleo bruto com grau American Petroleum Institute (API) de $28,3^{\circ}$. A água utilizada foi proveniente da Companhia Estadual de Águas e Esgotos do Rio de Janeiro (CEDAE), a concessionária de água do estado do Rio de Janeiro.

A concentração de óleo foi controlada por uma bomba dosadora do tipo cromatográfica, adotada pelo fato de propiciar grande precisão na dosagem.

O gradiente de velocidade da mistura da água com o óleo foi controlado através de uma válula do tipo agulha, dotada de medidores de pressão a montante e a jusante. O diâmetro de gotas de óleo desejado era fixado através da diferença de pressão entre esses medidores.

\section{A unidade experimental}

Os ensaios foram realizados em uma unidade experimental (Figura 1) constituída por um módulo de membranas de microfiltração do tipo fibra oca, implantado de forma submersa em um reservatório que era continuamente alimentado pelo efluente sintético descrito anteriormente.

A força motriz utilizada para promover a permeação da água através das membranas foi gerada por uma bomba hidráulica (B-1). Essa bomba foi também utilizada para promover um dos mecanismos usados para realização da limpeza física das membranas e, consequente, atenuação do fouling, a retrolavagem.

A alternância entre os modos de operação normal e de retrolavagem era realizada através da abertura e do fechamento de quatro válvulas solenoides (VS-1, VS-2, VS-3 e VS-4). Essas válvulas eram comandadas por um temporizador, através do qual os tempos de operação normal e de retrolavagem eram previamente fixados.

O material utilizado nas membranas foi produzido a partir da polieterimida. A área útil de permeação total das membranas foi de $0,5 \mathrm{~m}^{2}$. Os diâmetros de poros médio e máximo foram de 0,4 e $0,8 \mu \mathrm{m}$, respectivamente.

Além da retrolavagem, foi utilizado outro método de limpeza física: o borbulhamento de ar na base dos módulos das membranas.

\section{Limpeza das membranas}

Após a realização de cada ensaio, era realizada a limpeza química. $\mathrm{O}$ seu objetivo era recuperar a permeabilidade hidráulica das membranas, cujo valor original foi de 150 a $200 \mathrm{~L} \cdot \mathrm{h}^{-1} \cdot \mathrm{m}^{-2}$ bar. Os testes para determinação da permeabilidade foram realizados com água da CEDAE, citada previamente.

O procedimento utilizado para a limpeza foi o de imersão das membranas em uma solução de água e o produto químico comercialmente conhecido como Vanish ação $\mathrm{O}_{2}$, cujo principio ativo é o 
percarbonato de sódio. Após a limpeza, a permeabilidade hidráulica das membranas era medida. Caso o seu valor não estivesse dentro da faixa desejada, a limpeza era refeita.

\section{Análises de laboratório e coleta de dados}

Para controle do processo foram realizadas duas análises de laboratório: O\&G e determinação do diâmetro das gotas de óleo.

Os métodos de determinação de $\mathrm{O} \& \mathrm{G}$ usualmente adotados em laboratório utilizam trabalhosas etapas de extração prévia do óleo da emulsão com solventes orgânicos diversos, como o n-hexano (EBRAHIMI et al., 2010) e o tetracloreto de carbono (SOKOLOVIĆ; SOKOLOVIĆ; SEVIC, 2009). Para supererar esse problema neste trabalho, as análises de O\&G foram feitas através da turbidez, determinada através do turbidímetro portátil Hach 2100P. Para tanto, foi elaborada uma curva de correlação de vários $\mathrm{O} \& \mathrm{G}$ com valores conhecidos versus turbidez.

Para determinação do diâmetro de gotas foi utilizado o Malvern Master Sizer, cuja capacidade de medição de diâmetro médio de partículas é entre 0,02 e $2.000 \mathrm{~mm}$.

\section{Condições dos experimentos}

Foram realizados 15 experimentos. O tempo de uma corrida operacional de cada um durou entre 7 e 8 horas, com a retrolavagem sendo realizada a cada 15 minutos de operação e durante um tempo de 15 segundos.

As amostras utilizadas tinham diâmetros de gotas de óleo entre 4 e $8 \mu \mathrm{m}$.

Neste trabalho, os experimentos tiveram três objetivos. O primeiro visou avaliar a qualidade do efluente tratado para diferentes condições operacionais, envolvendo valores diferentes dos seguintes parâmetros: O\&Gam, Q, $\Delta$ p e Rec. Os valores dos parâmetros testados foram: O\&Gam variando entre 100 e $260 \mathrm{mg} . \mathrm{L}^{-1}, \Delta \mathrm{p}$ com valores de $-0,20$ e $-0,30$ bar, Q com valores iniciais de 30 e 60 L.h ${ }^{-1}$ e Rec com valores de 0,75 e 0,90. Para avaliação dos resultados dos ensaios, foram avaliadas a Em, em termos de remoção de óleo, e a O\&Gp.

O segundo objetivo dos experimentos foi avaliar a queda de Jp ao longo do tempo e o valor do Jp após o tempo de operação de 6 horas nas seguintes condições: $\Delta$ p fixado em - 0,20 bar; 2 valores de O\&Gam, 100 e $200 \mathrm{mg} . \mathrm{L}^{-1}$; e dois de Rec, 0,75 e 0,90 .

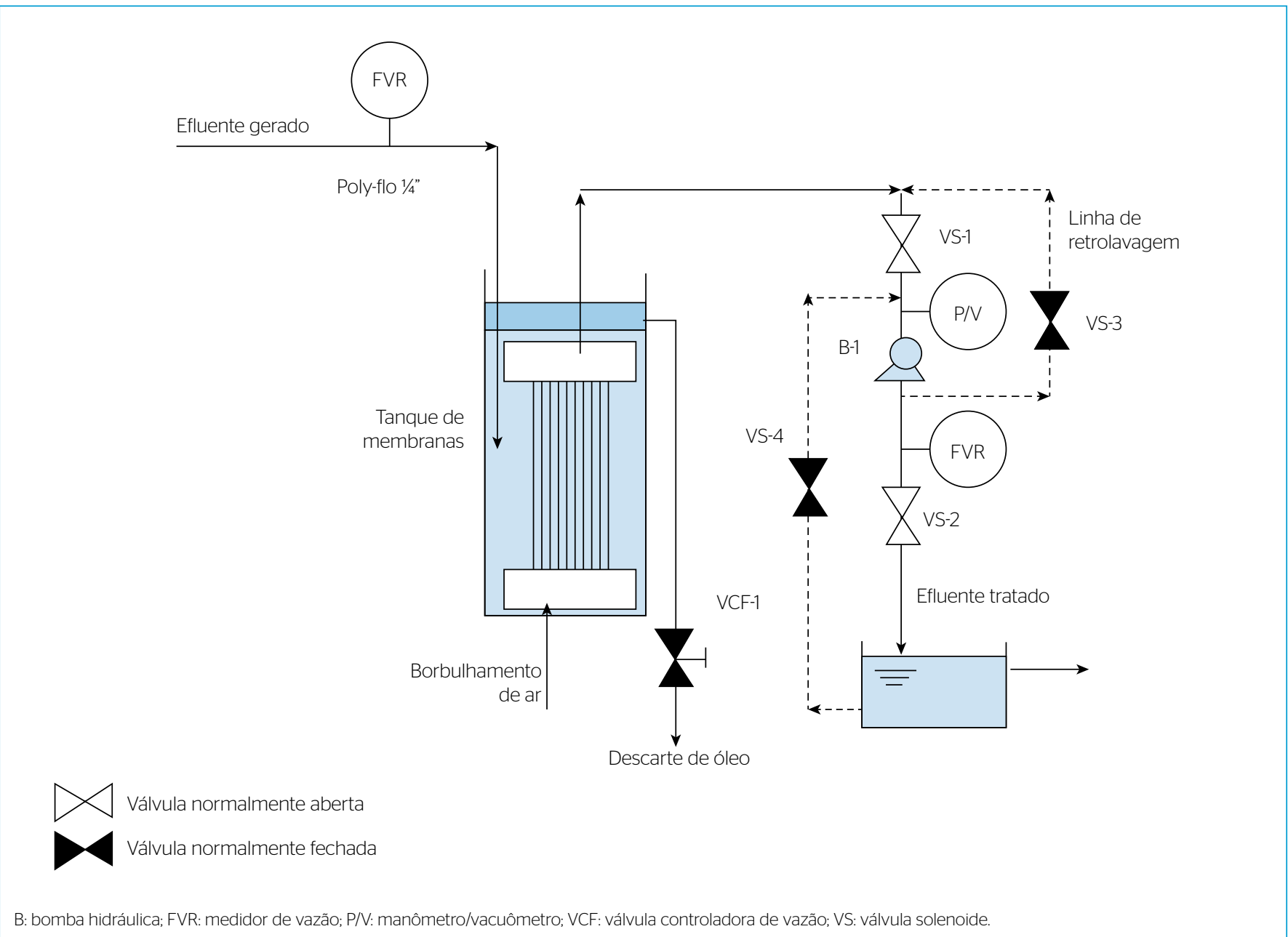

Figura 1 - Fluxograma da unidade experimental para o tratamento de efluentes oleosos através do processo de membranas. 
O terceiro objetivo dos experimentos foi avaliar a influência da variação dos valors de $\Delta \mathrm{p}$ no Jp, para diferentes valores de Q e Rec.

\section{RESULTADOS E DISCUSSÕES}

Os resultados estão apresentados a seguir.

\section{Qualidade do efluente tratado}

Os resultados da avaliação da qualidade do efluente tratado para diferentes condições operacionais estão apresentados na Figura 2.

De acodo com a Figura 2, os valores de O\&Gp encontrados foram relativamente baixos e resultaram em Em elevadas, com um valor mínimo de $92 \%$.

Dos 15 testes realizados, os valores de O\&Gp médio e máximo foram de 4,9 e 9,5 mg. $\mathrm{L}^{-1}$, respectivamente. Esses valores estão muito abaixo do que o fixado para $O \& G$ pela Resolução do Conselho Nacional do Meio Ambiente (CONAMA) no 357/2005 (BRASIL, 2005), atualizada na Resolução n 430/2011 (BRASIL, 2011), a fim de que o efluente possa ser descartado em corpos de água superficiais.

De fato, valores médios de O\&Gp tão baixos podem indicar a possibilidade de reúso do permeado em atividades industriais diversas, como lavagem de peças em oficinas mecânicas, lavagem de tubos de colunas de perfuração de poços de petróleo e lavagem de veículos industriais diversos, como os de terraplenagem ou locomotivas.

Um aspecto importante observado é que os valores de O\&Gp independem dos valores de O\&Gam, para a faixa de valores testados desse parâmetro (entre 100 e $260 \mathrm{mg.L}^{-1}$ ). Isso ocorreu para ambos os valores de pressão aplicados (-0,20 e -0,30 bar).

\section{Influência da concentração de óleos e graxas na alimentação das membranas e da taxa de recuperação de água no declínio de fluxo de permeado}

Conforme citado previamente, o segundo objetivo dos experimentos visou avaliar a queda de Jp ao longo do tempo e o valor desse parâmetro após seis horas de operação para diferentes condições operacionais.

Alguns parâmetros operacionais influenciam na queda de Jp, como é o caso do O\&Gam e do Rec. Na Figura 3 são apresentadas as curvas de queda de Jp normalizado (Jp/Jp-inicial), ao longo do tempo, para $\Delta \mathrm{p}$ de $-0,20$ e diferentes valores de O\&Gam e Rec.

De acordo com a Figura 3, as curvas dos ensaios 1 e 3 indicam que, para as mesmas Rec, o maior valor do O\&Gam (200 mg.L.-1) resulta em um maior declínio de fluxo. Isso ocorre pelo fato de um maior O\&Gam causar um maior O\&G na corrente de concentrado (O\&Gc), cujo valor

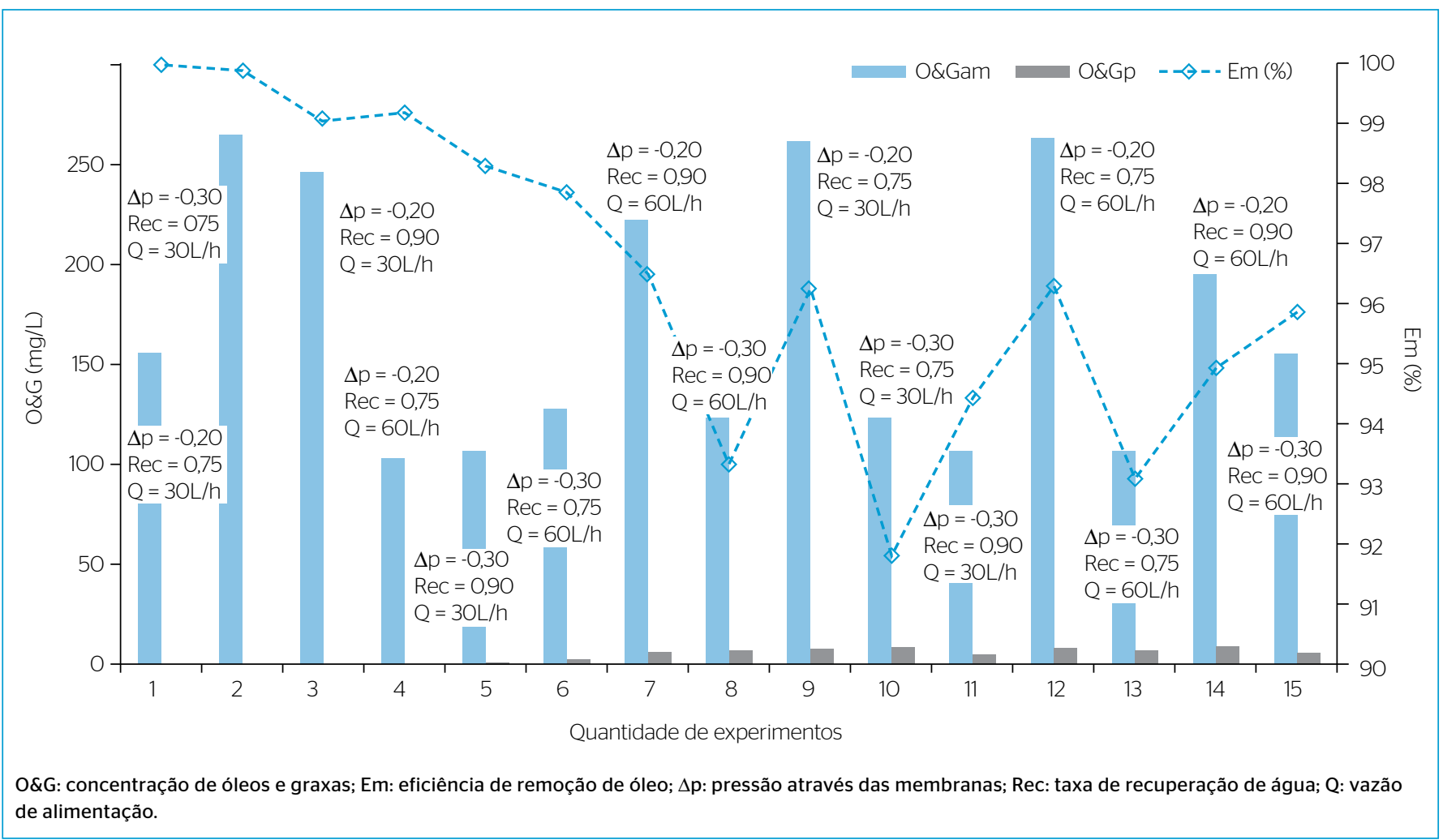

Figura 2 - Resultados dos valores de concentração de óleos e graxas no permeado e da eficiência de remoção de óleo das membranas para diferentes valores de concentração de óleos e graxas na alimentação das membranas. 
é igual ao do $\mathrm{O} \& \mathrm{G}$ do interior do tanque das membranas. Com o aumento do $\mathrm{O} \& \mathrm{Gc}$ há um aumento do $\mathrm{O} \& \mathrm{G}$ próximo à superfície da membrana, o que eleva a resistência ao transporte da água e do óleo, resultando em maiores retenções da fase oleosa. Assim, o processo de fouling ocorrerá de forma mais intensa.

O outro parâmetro que influencia na queda de Jp é o Rec. Ainda de acordo com a Figura 3, as curvas dos ensaios 2 e 3 indicam que, para as mesmas concentrações, o maior valor do $\operatorname{Rec}(0,90)$ resulta em um maior declínio de Jp. Isso ocorre pelas mesmas condições citadas no item anterior, no caso do O\&Gam. A elevação do Rec resulta em um maior O\&Gc, o que intensificará o processo de fouling.

Um aspecto importante a ser destacado é o comportamento do Jp ao longo do tempo. De acordo com a Figura 3, observa-se que o declínio do Jp ocorre sob a forma de dois estágios. No primeiro, o declínio ocorre de forma bastante acentuada, fato que ocorre apenas nas primeiras horas do ensaio. No segundo estágio o declínio ocorre de forma mais suave, tornando-se quase constante com o tempo, o que perdura até o restante do ensaio.

As possíveis causas do declínio conforme o primeiro estágio são a predominância de ocorrência de bloqueio dos poros e a polarização de concentração sobre a formação de torta (de gotas de óleo, no caso) sobre a superfície das membranas (OHYA, 1998).

\section{Influência da pressão através das membranas no fluxo de permeado}

A elevação da $\Delta$ p causa elevação do Jp, já que a força motriz para o processo aumenta. Entretanto, há um determinado valor de $\Delta \mathrm{p}$ a partir do qual uma elevação adicional desse parâmetro não resulta em elevação adicional de fluxo. Esse fluxo máximo é chamado de fluxo limite (MULDER, 1996).

Os resultados da influência do $\Delta \mathrm{p}$ sobre o Jp estão apresentados na Figura 4. Conforme citado previamente, os valores de Jp foram considerados para seis horas. Um aspecto importante a ser destacado é que o tempo de 6 horas está dentro do citado segundo estágio do declínio de fluxo, onde a sua queda ocorre de forma mais suave com o tempo.

De acordo com o gráfico, mantidas as mesmas condições, todos os ensaios realizados com o maior valor de $\Delta \mathrm{p}$ (-0,30 bar) apresentaram valores de Jp maiores do que os realizados com o $\Delta \mathrm{pm}$ de -0,20. Isso ocorreu independentemente dos valores de O\&Gam e dos dois valores de Rec testados $(0,75$ e 0,90$)$.

\section{CONCLUSÕES}

Entre os aspectos mais importantes observados no trabalho está o de que as membranas de microfiltração produzem um efluente final com

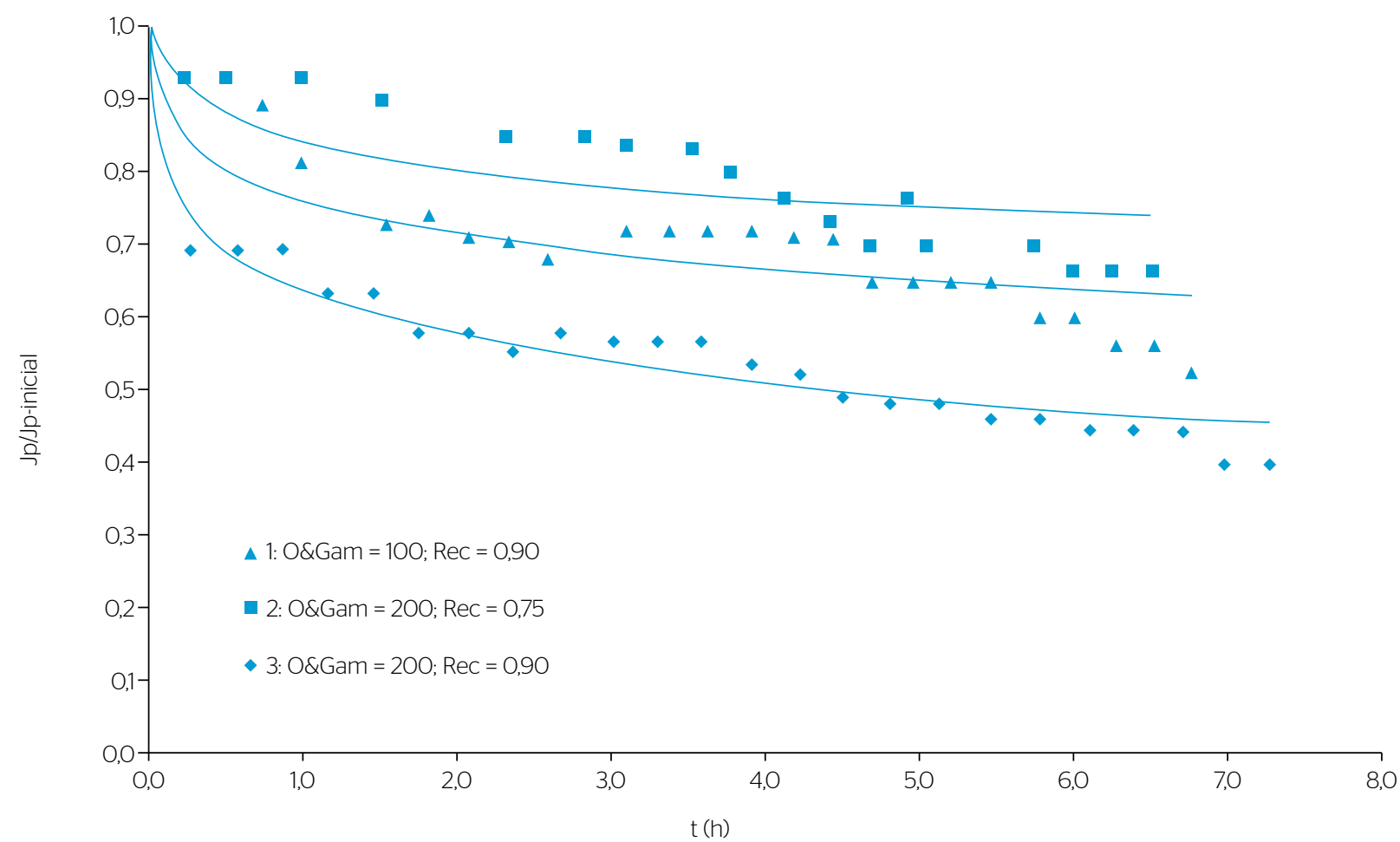

Jp/Jp-inicial: fluxo de permeado normalizado; O\&Gam: concentração de óleos e graxas na alimentação das membranas; Rec: taxa de recuperação de água.

Figura 3 - Evolução da queda de fluxo de permeado ao longo do tempo para pressão através das membranas com valor de -0, 20 bar e valores de concentração de óleos e graxas na alimentação das membranas de 100 e $200 \mathrm{mg} \cdot \mathrm{L}^{-1}$ e taxas de recuperação de água de 0,75 e 0,90. 


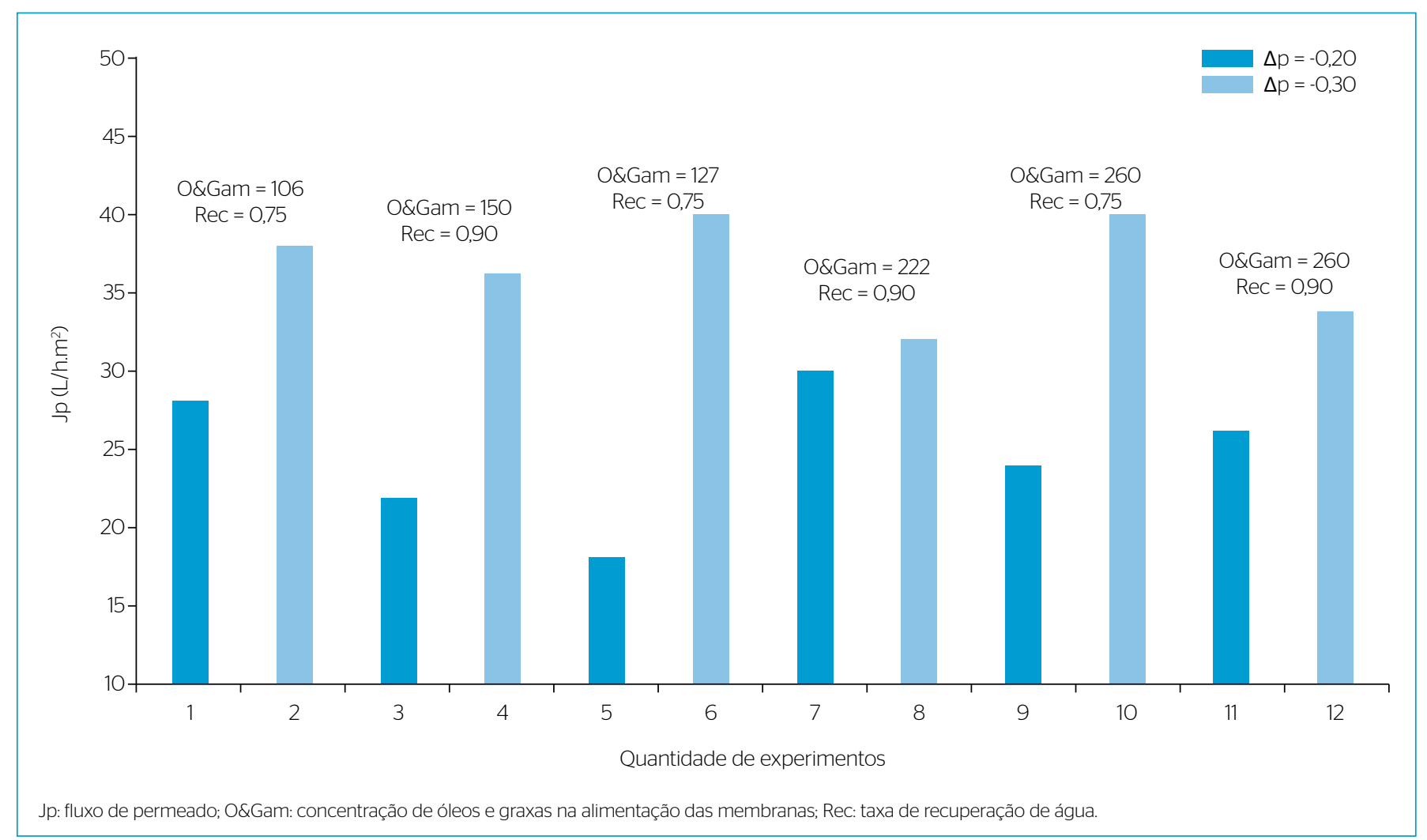

Figura 4 - Comparação entre os valores do fluxo de permeado após seis horas de andamento do ensaio para valores de $\Delta p$ de $-0,20$ e $-0,30$ bar, para diferentes valores de concentração de óleos e graxas na alimentação das membranas e valores de taxa de recuperação de água de 0,75 e 0,90.

boa qualidade, a ponto de possibilitar o seu reúso em atividades industriais diversas. Dos 15 testes realizados, os valores de O\&Gp médio e máximo foram de 4,9 e 9,5 mg.L.-1, respectivamente.

Outro aspecto relevante observado foi a influência de alguns parâmetros no declínio do Jp. Entre eles estão o O\&Gam e a Rec. A elevação de ambos os parâmetros acentua o declínio de Jp de modo significativo.

Verificou-se ainda que outro parâmetro importante no processo é a $\Delta \mathrm{p}$. Neste trabalho, verificou-se que, se mantidas as mesmas condições, os ensaios realizados com o maior valor de $\Delta \mathrm{p}$ testado (-0,30 bar) apresentaram maiores de valores de Jp para o tempo de seis horas, fato que ocorreu para diversos valores de O\&Gam e para os dois valores de Rec testados.

\section{FONTE DE FINANCIAMENTO}

Universidade Federal do Rio de Janeiro (UFRJ)/Instituto Alberto Luiz Coimbra de Pós-Graduação e Pesquisa de Engenharia (Coppe), Petrobras, Universidade Federal da Bahia (UFBA).

\section{REFERÊNCIAS}

AL-OBEIDANI, S.K.S.; AL-HINAI, H.; GOOSEN, M.F.A.; SABLANI, S.; TANIGUCHI, Y.; OKAMURA, H. (2008) Chemical cleaning of oil contaminated polyethylene hollow fiber microfiltration membranes. Journal of Membrane Science, Amsterdã, v. 307, n. 2, p. 299-308. https://doi.org/10.1016/j. memsci.2007.09.048

BADER, M.S.H. (2007) Seawater versus produced water in oilfields water injection operations, Desalination, v. 208, n. 1-3, p. 159-168. https://doi.org/10.1016/j.desal.2006.05.024
BRASIL. (2005) Conselho Nacional de Meio Ambiente. Resolução no 357 - 17 mar. 2005. Dispõe sobre a classificação dos corpos de água e diretrizes ambientais para o seu enquadramento, bem como estabelece as condições e padrões de lançamento de efluentes, e dá outras providências. Diário Oficial da União, Brasília.

BRASIL. (2011) Conselho Nacional de Meio Ambiente. Resolução no 430, de 13 de maio de 2011. Dispõe sobre as condições e padrões de lançamento de efluentes, complementa e altera a Resolução no 357, de 17 de março de 2005, do Conselho Nacional do Meio Ambiente-CONAMA. Diário Oficial da União, Brasília. 
CAMBIELLA, À.; ORTEA, E.; RÍOS, G.; BENITO, J.M.; PAZOS, C.; COCA, J. (2006) Treatment of oil-in-water emulsions: Performance of a sawdust bed filter. Journal of Hazardous Materials, v. 131, n. 1-3, p. 195-199. https://doi.org/10.1016/j.jhazmat.2005.09.023

CHAKRABARTY, B.; GHOSHAL, A.K.; PURKAIT, M.K. (2008) Ultrafiltration of stable oil-in-water emulsion by polysulfone membrane. Journal of Membrane Science, v. 325, n. 1, p. 427-437. https://doi.org/10.1016/j.memsci.2008.08.007

EBRAHIMI, M.; ASHAGHI, K.S. ; WILLERHAUSEN, D. ; MUND, P. ; BOLDUAN, P. ; CZERMAK, P. (2009) Characterization and application of different ceramic membranes for the oil-field produced water treatment. Desalination, v. 245, n. 1-3, p. 533-540. https://doi.org/10.1016/j.desal.2009.02.017

EBRAHIMI, M.; WILLERHAUSEN, D. ; ASHAGHI, K.S. ; ENGEL, L. ; PLACIDO, L. ; MUND, P. ; BOLDUAN, P. ; CZERMAK, P. (2010) Investigations on the use of different ceramic membranes for efficient oil-field produced water treatment. Desalination, v. 250, n. 3, p. 991-996. http://dx.doi.org/10.1016/j.desal.2009.09.088

HONG, A.; FANE, A.G.; BURFORD, R. (2003) Factors affecting membrane coalescence of stable oil-in-water emulsions. Journal of Membrane Science, v. 222, n. 1-2, p. 19-39. https://doi.org/10.1016/ s0376-7388(03)00137-6

MAITI, S.; MISHRA, I.M.; BHATTACHARYA, S.D.; JOSHI, J.K. (2O11) Removal of oil from oil-in-water emulsion using a packed bed of commercial resin. Colloids and Surfaces A: Physicochemical and
Engineering Aspects, v. 389, n. 1, p. 291-298. http://dx.doi.org/10.1016/j. colsurfa.2011.07.041

MATHAVAN, G.N.; VIRARAGHAVAN, T. (1992) Coalescence/Filtration of a oil-in-water emulsion in a peat bed. Water Research, v. 26, n. 1, p. 91-98. https://doi.org/10.1016/0043-1354(92)90116-L

MULDER, M. (1996) Basic principles of membrane technology. 2. ed. Dordrecht: Kluwer Academic. 564 p.

OHYA, H.; KIM, J.J.; CHINEN, A.; AIHARA, M.; SEMENOVA, S.I; NEGISHI, Y.; MORI, O.; YASUDA, M. (1998) Effects of pore size on separation mechanisms of microfiltration of oily water, using porous glass tubular membrane. Journal of Membrane Science, v. 145, n. 1, p. 1-14. https://doi.org/10.1016/S0376-7388(98)00067-2

SAIDI, M.; MADDAHIAN, R.; FARHANIEH, B.; AFSHIN, H. (2O12) Modeling of flow field and separation efficiency of a deoiling hydrocyclone using large eddy simulation. International Journal of Mineral Processing, v. 112-113, p. 84-93. https://doi.org/10.1016/j. minpro.2012.06.002

SOKOLOVIĆ, R.M.S.; SOKOLOVIĆ, S.M; SEVIC, S. (2009) Oily water treatment using a new steady-state fiber-bed coalescer. Journal of Hazardous Materials, v. 162, n. 1, p. 410-415. https://doi.org/10.1016/j. jhazmat.2008.05.054

STEWART, M.; ARNOLD, K. (2011) Part 1 - Produced Water Treating Systems. In: STEWART, M.; ARNOLD, K. Produced Water Treatment Field Manual. Amsterdã: Elsevier. p. 1-134. 